\title{
Complete Genome Sequence of a Novel Reassortant H11N2 Avian Influenza Virus Isolated from a Live Poultry Market in Eastern China
}

\author{
Yuee Zhang, Qiaoyang Teng, Chaochao Ren, Guoxin Li, Xuesong Li, and Zejun Li \\ Department of Avian Infectious Disease, Shanghai Veterinary Research Institute, Chinese Academy of Agricultural Sciences, Shanghai, China
}

A/chicken/Nanjing/908/2009(H11N2) (CK908) was isolated from a live poultry market in Nanjing, China. Using PCR and sequencing analysis, we obtained the complete genome sequences of the CK908 virus. The sequence analysis demonstrated that this $\mathrm{H11N2}$ virus was a novel reassortant AIV whose PB1, PB2, PA, HA, NP, NA, M, and NS genes originated from H9N2, H7N7, $\mathrm{H} 5 \mathrm{~N} 2, \mathrm{H} 11 \mathrm{~N} 8, \mathrm{H} 3 \mathrm{~N} 6, \mathrm{H} 6 \mathrm{~N} 2, \mathrm{H} 1 \mathrm{~N} 1$, and H5N1, respectively. Knowledge regarding the complete genome sequences of the CK908 virus will be useful for epidemiological surveillance.

\begin{abstract}
$\Lambda$ vian influenza virus (AIV) originates from birds, particularly A wild waterfowl, and has been demonstrated to have 16 hemagglutinin (HA) and 9 neuraminidase (NA) subtypes $(1,3)$. In the early 1970s, live poultry markets (LPM) were considered to be a prolific source of AIVs (5). H11N2 subtype avian influenza virus can infect wild birds (1), turkey, and gull (3). In addition, serum surveillance demonstrated that Lebanese backyard poultry growers, who were frequently exposed to chicken, may have been infected with H11 avian influenza virus (4). This result indicated that $\mathrm{H} 11$ influenza virus may possess the ability to cross the species barrier to infect humans. Thus, it is important to enhance surveillance for H11 AIVs.
\end{abstract}

In this study, A/chicken/Nanjing/908/2009(H11N2) (CK908) was isolated from LPM in Nanjing, China. The PCR was performed using universal primers for influenza A virus (2). The amplification products were directly sequenced, and the SEQMAN program (DNASTAR, Madison, WI) was used to analyze the sequences.

The genome of the CK908 virus consisted of 8 segments of single-stranded RNA and was 13,623 nucleotides in length. The polymerase genes $\mathrm{PB} 1$ and $\mathrm{PB} 2$ each consisted of 2,341 nucleotides; the polymerase acidic (PA) gene consisted of 2,233 nucleotides; the HA gene, 1,760 nucleotides; the nucleoprotein (NP) gene, 1,565 nucleotides; the NA gene, 1,466 nucleotides; the matrix (M) gene, 1,027 nucleotides; and the nonstructural (NS) gene, 890 nucleotides.

Sequence analysis revealed that the nucleotide sequences of the HA gene of the CK908 virus were most closely related to that of the H11N8 isolate A/mallard/Netherlands/17/2007(H11N8) and shared 98\% nucleotide homology. The NA fragment was most closely related to $\mathrm{H} 6 \mathrm{~N} 2$ isolate A/mallard/Jiangxi/8264/2004 (H6N2) and shared 98\% nucleotide homology with it. The nucleotide sequences of PB1 and PB2 genes were most closely related to those of the H9N2 isolate A/avian/Egypt/920431/2006(H9N2) and the H7N7 isolate A/shoveler/Italy/2698-3/2006(H7N7), with which they shared $97 \%$ and $98 \%$ nucleotide homology, respectively. The nucleotide sequences of the PA gene were most closely related to that of the $\mathrm{H} 5 \mathrm{~N} 2$ isolate $\mathrm{A} /$ wild bird/Korea/A81/ 2009(H5N2) and shared 99\% nucleotide homology with it. The nucleotide sequences of the NP and M genes were most closely related to those of the $\mathrm{H} 3 \mathrm{~N} 6$ isolate $\mathrm{A} / \mathrm{red}$ crested pochard/
Mongolia/1915/2006(H3N6) and H1N1 isolate A/duck/Eastern China/103/2003(H1N1), with which they shared $98 \%$ and $95 \%$ nucleotide homology, respectively. The nucleotide sequences of the NS gene were most closely related to $\mathrm{H} 5 \mathrm{~N} 1$ isolate $\mathrm{A} /$ environment/Hunan/6-69/2008(H5N1) and shared 98\% nucleotide homology with it.

In conclusion, the CK908 virus isolate proved to be a novel reassortant AIV whose $\mathrm{PB} 1, \mathrm{~PB} 2, \mathrm{PA}, \mathrm{HA}, \mathrm{NP}, \mathrm{NA}, \mathrm{M}$, and NS genes originated from H9N2, H7N7, H5N2, H11N8, H3N6, $\mathrm{H} 6 \mathrm{~N} 2, \mathrm{H} 1 \mathrm{~N} 1$, and $\mathrm{H} 5 \mathrm{~N} 1$, respectively.

Nucleotide sequence accession numbers. The genome sequences of A/chicken/Nanjing/908/2009(H11N2) have been deposited in GenBank under accession numbers JX499146 to JX499153.

\section{ACKNOWLEDGMENTS}

This study was supported by the National Natural Science Foundation of China (no. 31172332), the Special Fund for Agro-scientific Research in the Public Interest, China (no. 201003012), the Special Fund for International Communication and Cooperation (no. S2011ZR0429), and the National High Technology Research and Development Program of China (863 Program) (no. 2011AA10A200).

\section{REFERENCES}

1. Henriques AM, et al. 2011. Multiyear surveillance of influenza A virus in wild birds in Portugal. Avian Pathol. 40:597-602.

2. Hoffmann E, Stech J, Guan Y, Webster RG, Perez DR. 2001. Universal primer set for the full-length amplification of all influenza A viruses. Arch. Virol. 146:2275-2289.

3. Karamendin K, et al. 2011. Phylogenetic analysis of avian influenza viruses of H11 subtype isolated in Kazakhstan. Virus Genes 43:46-54.

4. Kayali G, et al. 2011. Evidence of infection with H4 and H11 avian influenza viruses among Lebanese chicken growers. PLoS One 6:e26818. doi: 10.1371/journal.pone.0026818.

5. Shortridge KF. 1992. Pandemic influenza: a zoonosis? Semin. Respir. Infect. 7:11-25.

Received 20 August 2012 Accepted 20 August 2012 Address correspondence to Zejun Li, lizejun@shvri.ac.cn. Copyright @ 2012, American Society for Microbiology. All Rights Reserved. doi:10.1128/JVI.02236-12 